\title{
ORCID: UN SISTEMA GLOBAL PARA LA IDENTIFICACIÓN DE INVESTIGADORES
}

Consol García-Gómez

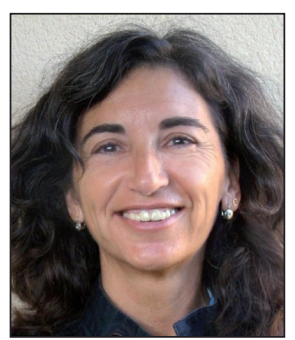

Consol García-Gómez es licenciada en filología española y diplomada en biblioteconomía y documentación por la Universidad de Barcelona en 1992. Desde 1997 trabaja en la Universidad Politécnica de Catalunya donde es responsable de los Servicios para la Investigación en la biblioteca del Campus del Baix Llobregat. Sus intereses son: el acceso abierto, los datos de investigación y los modelos de negocio de la comunicación científica. Es miembro de American Society for Engineering Education, de Optical Society of America y desde 2011 miembro de Orcid Outreach Working Group.

Universitat Politècnica de Catalunya (UPC), Servei de Biblioteques i Documentació Campus del Baix Llobregat. Esteve Terrades, 10. Edif D7 (Serveis Campus), pl. 2 08860 Castelldefels, Barcelona, España consol.garcia@gmail.com

\section{Resumen}

Se describe el proyecto Open Researcher and ContributorID (Orcid) que intenta resolver el problema de la identificación, ambigüedad y duplicidad en los nombres de los investigadores (autores y colaboradores) mediante la creación de un registro único. Éste estará conectado con otros sistemas actuales de identificación de autor como Author Resolver, Inspire, IraLIS, RePEc, ResearcherID, Scopus Author Identifier y VIVO, entre otros. Orcid se vinculará a la producción de los investigadores facilitando conocer sus publicaciones, identificando colaboradores y revisores y en definitiva, favoreciendo el proceso de descubrimiento científico.

\section{Palabras clave}

Comunicación científica, Identificadores de autor, Desambiguación, Registros de investigadores, Perfiles de autor.

\section{Orcid: A global system for authors identification in scholarly communication}

\begin{abstract}
Description of the project Open Researcher and ContributorID (Orcid) that tries to solve the name's ambiguity and duplicity problems to identify correctly the researchers (authors and contributors) by creating a single record. This record will be connected with other current systems of author identification as Author Resolver, Inspire, IraLIS, RePEc, Scopus Author Identifier, ResearcherID, VIVO and others. Orcid will be linked to the researchers publications to facilitate its visibility, identifying contributors and reviewers and, ultimately, promoting the process of scientific discovery.
\end{abstract}

\section{Keywords}

Scholarly communication, Author identifiers, Contributors, Author disambiguation, Author profiles.

García-Gómez, Consol. "Orcid: un sistema global para la identificación de investigadores". El profesional de la información, 2012, marzo-abril, v. 21, n. 2, pp. 210-212.

\section{Sistemas de identificación de autor}

Basándose en datos de Scopus, Takeda (2011) estima que hay algo más de 27 millones de investigadores trabajando en centros privados, públicos y universidades. Muchos de ellos son difíciles de identificar a través de sus trabajos ya sea porque no han firmado siempre igual, tienen nombres comunes, los cambian a lo largo del tiempo (por ej., en algunos países las mujeres al casarse) o porque los editores y productores de bases de datos los interpretan mal. Esto ocasiona problemas para el investigador-autor a la hora de recopilar su bibliografía y para el investigador-usuario en su búsqueda de referencias para su trabajo.

Existen diferentes sistemas de identificación de autor: Author Resolver, Inspire, IraLIS, RePEC, Scopus Author Identifier, ResearcherID, VIVO y otros, pero hasta ahora ninguno goza de una aplicación universal.

Nos podemos preguntar por qué se ha tardado tanto tiempo en acordar un identificador o DAI (digital author identifier) [por ejemplo a la manera del conocido DOI (digital object identifier) para identificar artículos y otros objetos digitales, 


\section{ORCID $\begin{aligned} & \text { Open Researcher } \\ & \text { \&Contributor ID }\end{aligned}$}

puesto en marcha en 2000] que identifique unívocamente a todos los autores.

Una de las razones del retraso ha sido la complejidad de su implementación (Fenner, 2011), pues no se quería tener un simple número, sino un sistema con el que además cada autor disponga de un espacio en el que pueda registrar sus datos y sus trabajos, y si lo desea, compartirlos. Y también un sistema de claims (reivindicaciones) en el que los autores puedan afirmar o declarar públicamente que una obra concreta es suya.

\section{El proyecto Orcid}

Orcid, organización sin ánimo de lucro, nació a finales del año 2009 a propuesta del Nature Publishing Group y de Thomson Reuters con el objetivo de crear un identificador de autores de publicaciones científicas, entendiendo autor y publicaciones científicas en término amplio: todo creador de contenido científico y todo tipo de documento científico. El investigador ha de ser reconocido por cualquier aportación que haga a la ciencia, sea un artículo en una revista o un dataset en un repositorio. Los identificadores, que deben ser permanentes y claros, permitirán la atribución de las obras a sus autores, los cuales podrán crear y mantener sus perfiles académicos de forma gratuita (Orcid principles).

Orcid será beneficioso para toda la comunidad científica: autores, universidades, agencias de financiación y editores

La diversidad de los más de 300 miembros de Orcid [entre los cuales se encuentra esta revista El profesional de la información], refleja los intereses de los diferentes agentes implicados en la publicación científica: autores, editores, universidades, sociedades profesionales y agencias gubernamentales. Sin embargo, desde su creación siempre ha habido un colectivo mayoritario: el sector académico, que a día de hoy representa un $42 \%$.

Los beneficios de Orcid para el investigador se manifiestan en 3 momentos de su vida profesional que se corresponden con los 3 principales agentes implicados en la iniciativa:

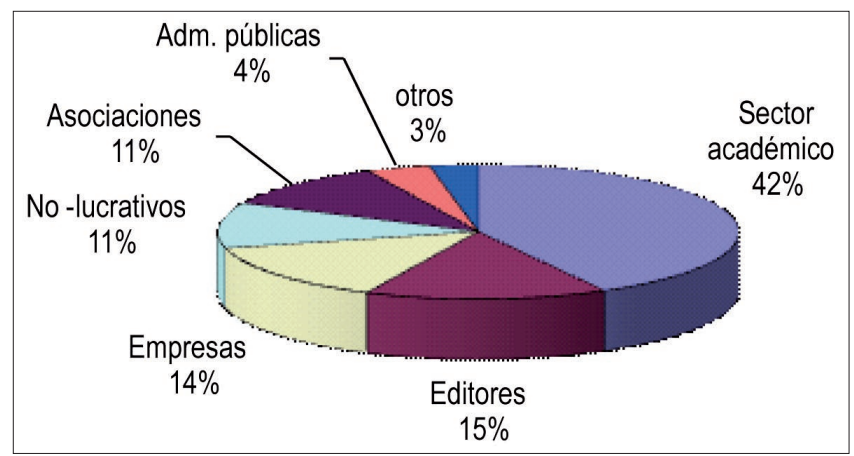

Figura 1. Tipos de miembros de Orcid
- el sector académico, cuando los investigadores acceden a la universidad o centro de investigación;

- las agencias de financiación, cuando solicitan proyectos y becas; $y$

- los editores de revistas científicas, cuando publican los resultados de su trabajo.

A las instituciones les ayudará en el proceso de evaluación de sus investigadores, pues podrán conocer exactamente la producción científica de cada uno; a los editores les servirá para mejorar la comunicación con los autores, incluido el proceso de peer review; y a las agencias de financiación les servirá para agilizar el proceso de presentación de propuestas y para saber qué ha pasado con la investigación que han financiado (Bilder, 2011).

\section{El investigador y Orcid}

Una vez el autor se haya registrado, podrá editar, modificar, actualizar su registro y tendrá todo el control sobre qué datos de su perfil quiere que sean públicos o privados, o compartirlos sólo con determinados agentes (editores, universidades, etc.). Los datos privados sólo serán usados por el sistema para hacer desambiguaciones en casos de duda.

Ya se ha dicho que Orcid será un sistema global dada su naturaleza "inclusiva" para con el resto de identificadores de autor de la escena científica. Otra característica importante es que será un sistema híbrido en cuanto a las afirmaciones para determinar la identidad de los autores. De esta forma no sólo será el autor el que podrá afirmar sus autorías, sino que las afirmaciones podrán proceder de instituciones e incluso del entorno social (LinkedIn). Un autor por ejemplo podrá reclamar un artículo como suyo, una institución podrá validar que tal autor está afiliado a la misma, y un editor podrá afirmar que es revisor de una determinada revista (Bilder, 2011). El hecho de que las afirmaciones tengan procedencias diversas y que pueda haber más de una afirmación de cualquier tipo para un único registro Orcid, implicará que inevitablemente haya registros repetidos y de ahí que se esté trabajando en mecanismos de desambiguación.

Serán posibles las siguientes consultas:

\begin{tabular}{|l|l|}
\hline Nombre & \multicolumn{1}{|c|}{ Descripción } \\
\hline Bio & Nombre y datos de afiliación de un autor dado \\
\hline Works & Trabajos de un autor dado \\
\hline Full & $\begin{array}{l}\text { Nombre y orcid de un autor, datos de afiliación y lista de } \\
\text { sus trabajos }\end{array}$ \\
\hline Work & Autores (con sus orcids) de un trabajo dado \\
\hline Search & $\begin{array}{l}\text { Lista de posibles autores (y sus orcids) a partir de los } \\
\text { metadatos conocidos o disponibles }\end{array}$ \\
\hline
\end{tabular}

\section{Implementación}

Para que el sistema funcione será necesario que acumule una gran masa crítica de registros, pero la relevancia y procedencia de los miembros de su junta directiva (Thomson Reuters, Elsevier, Wiley-Blackwell, Nature Publishing Group, Harvard, Cornell, MIT, Medizinische Hochschule Hannover, Inspire, CERN, CrossRef, ACM, OCLC, Wellcome Trust, National Institute of Informatics...) permite albergar sólidas 
esperanzas de éxito. Para su financiación Orcid ha logrado recaudar 2 millones de dólares en 2011.

No va a haber una agencia de registro para los identificadores, como la hay para los DOIs (CrossRef), y tampoco los autores tendrán que pagar para crear un registro. Orcid es una iniciativa sin ánimo de lucro e independiente, con diferentes stakeholders o partes interesadas, no limitada a un área geográfica ni temática, transparente y basada en código abierto.

La base de partida de Orcid será el sistema ResearcherID, de Thomson Reuters, empresa que ha cedido el software. Se anticipa que el identificador orcid tendrá una estructura alfanumérica parecida a los ResearcherID, pero se tratará de un identificador global e independiente, no ligado a ninguna editorial. Los registros se vincularán a los otros sistemas de identificación de autor. Así por ejemplo ya se ha visto su compatibilidad con VIVO, muy extendido en Estados Unidos y poco a poco se mapeará a los otros identificadores. Sin embargo se pretende que Orcid sea el destino final de todos ellos. Los orcids se expresarán como urls, por ejemplo (ficticio): http://orcid.org/A-1963-7688

\section{Además de contener los datos propios,} los registros de Orcid estarán conectados con otros sistemas de identificación de autor

2011 fue clave para el proyecto: como se ha dicho, Thomson Reuters cedió gratuitamente el software, se diseñó el sistema, y se acordó su modelo de negocio. A finales de año se contrató a Semantico para adaptar el software y se lanzó la fase 1 que se espera concluya en el primer trimestre de 2012. Esta fase se caracteriza por centrarse en investigadores en activo; tener en cuenta la información y las afirmaciones procedentes de autores e instituciones, y solucionar problemas de ambigüedad.

En la fase 2 se recaudarán las cuotas a las instituciones, que serán en función de su tamaño, y en el último semestre de 2012 se recogerán las afirmaciones de los editores, y se buscarán mecanismos para solucionar la duplicidad de registros. El sistema estará listo para su lanzamiento para 2013.

\section{Conclusiones}

Orcid es mucho más que un mecanismo de enlaces entre diferentes sistemas de identificación: se trata de un sistema global, abierto (en información y en software) e integrador (de universidades, agencias de financiación, editores) con contenido propio (Brand, 2011). El primer servicio que se pondrá en marcha es la identificación y desambiguación de los autores, pero luego ofrecerá nuevos servicios (por ejemplo ayuda para elaborar los curriculum vitae), según las necesidades de las partes implicadas, entre ellas las de los editores.

En febrero de 2012 las instituciones españolas miembros de Orcid son sólo estas 9 (marzo 2012): Colegio Oficial de Psicólogos de Madrid, Dialnet, El profesional de la información (EPI), Fundación Ciudad de la Energía, iMedPub, International Registry for Authors-Links to Identify Scientists (IraLIS), Servei de Biblioteques de la Univ. Politècnica de Catalunya, Univ. de
Cádiz. y Univ. del País Vasco. Por la magnitud del proyecto y los beneficios que puede reportar a la comunidad científica merecería más atención. Sería conveniente que alguna organización española liderara su extensión en España.

\section{Bibliografía}

Aerts, Raf. "Digital identifiers work for articles, so why not for authors?". Nature, 2008, v. 453, n. 7198, Jun, p. 979. http://dx.doi.org/10.1038/453979b

Bilder, Geoffrey. "Disambiguation with de-duplication: Modeling authority and trust in the Orcid system". March 2011.

http://orcid.org/sites/default/files/disambiguation-dedupli cation_wp_v4.pdf

Bilder, Geoffrey. "Structure of the Orcid specification", 2011, v. 2, 12th Dec.

http://about.orcid.org/content/structure-orcid-identifierspecification

Bilder, Geoffrey; Wilson, Bryan. What Orcid already does and what Orcid can do? September 16, 2011.

http://about.orcid.org/content/what-orcid-already-does\% E2\%80\%A6-and-what-comes-next

Brand, Amy; Bilder, Geoffrey; Warner, Simeon. "Orcid Technical Update" CNI Fall Membership Meeting, 13 Dec 2011. http://www.cni.org/wp-content/uploads/2011/12/cni_ orcid_bilder.pdf

Fenner, Martin. Author identifier overview, Jan. 2nd 2011. http://blogs.plos.org/mfenner/author-identifier-overview

Fenner, Martin. "Orcid or how to build a unique identifier for scientists in 10 easy steps". Gobbledygook Blog, 2010. http://blogs.plos.org/mfenner

Fenner, Martin; García-Gómez, Consol; Thorisson, Gudmundur A. "Collective action for the Open researcher \& contributor ID (Orcid)". Serials: The journal for the serials community, 2011, v. 24, n. 3, Nov., pp. 277-279. http://dx.doi.org/10.1629/24277

Habibzadeh, Farrokh; Yadollahie, Mahboobeh. "The problem of 'Who'". The international information \& library review, 2009, v. 41, n. 2, Jun., pp. 61-62.

http://dx.doi.org/10.1016/j.iilr.2009.02.001

http://www.sciencedirect.com/science/article/pii/ S1057231709000095

Nature. "Credit where credit is due" [editorial]. Nature, 2009, 17th Dec., v. 462, p. 825.

http://dx.doi.org/10.1038/462825a

http://www.nature.com/nature/journal/v462/n7275/full/ 462825a.html

Orcid. Mission statement.

http://orcid.org/mission-statement

Orcid. Principles.

http://www.orcid.org/principles

Takeda, Hidekai. "An introduction to Orcid". Coinfo conf., Hangzhou, China, Dec. 22, 2011.

http://orcid.org/content/introduction-orcid 